\title{
Impact of relaying on inter-cell interference in mobile cellular networks
}

\author{
K. Georgiev \\ Technical University of Sofia, \\ Bulgaria \\ E-mail: kamen.georgiev@yahoo.com
}

\author{
D. C. Dimitrova \\ University of Twente, The Netherlands \\ E-mail: d.c.dimitrova@ewi.utwente.nl
}

\begin{abstract}
Incorporating relay nodes in cellular networks, e.g. UMTS/ HSPA, is beneficial for extending coverage as well as for service enhancement. In this paper we study the inter-cell interference generated by a relay-enabled cell and how this influences the performance of mobile users. The performance measures of interest are the inter-cell interference distribution, realized rates and flow throughput. Our investigations show that not only relaying reduces interference but as result of this decrease all users experience an additional performance improvement, independently whether they use a relay or not. The effect is even stronger when flow throughputs are evaluated. The consideration of flow dynamics is a strong and distinctive aspect of our analysis methodology. We show that the evaluation on flow level contributes significantly to better understanding of the effects of relaying. Special attention is also given to the applied scheduling scheme.
\end{abstract}

\section{INTRODUCTION}

In the last decade the number of mobile subscribers has increased dramatically. Combined with the ever growing number of mobile applications, the demands towards system capacity increase. This phenomenon exhibits, as expected, on the downlink (from base station to mobile) but also on the uplink (from mobile to base station). In order to provide enhanced capacity, operators first deployed UMTS (Universal Mobile Telecommunications System) cellular networks and subsequently upgraded them with the HSPA (High Speed Packet Access) technologies - HSDPA (High-Speed Downlink Packet Access) for the downlink, see [1], and EUL (Enhanced UpLink) for the uplink, see [2]. HSPA provides improved flexibility in matching user traffic requirements to the available channel resource. Despite all technological advances a special group of mobile users, i.e. users at the cell edge, continue to experience poor service due to strong signal degradation. These users are also very sensitive to the presence of interference, e.g. inter-cell interference, because it reduces the achievable SINR (Signal to Interference and Noise Ratio) at the base station.

Relaying is typically considered either to improve coverage, see [3], [4], or to increase system capacity, see [5], [6]. Indeed in [5] the authors show that on EUL for UMTS the data rates realized at the cell edge significantly improve when a relay is used. More importantly, the study shows that the flow throughput of all users rises independently whether they use a relay or not. This phenomenon cold not be observed if only data rates are evaluated thus stressing on the importance of modelling user dynamics, i.e. the change in number of active flows.

Many studies are dedicated to the performance benefits of relaying in WiMAX, e.g. [9], [10], or LTE networks, e.g. [3], [4], [11], [12]. However, up to our knowledge no study discusses the combined effects of relaying and scheduling on the inter-cell interference and the consequences of that on MS's performance. We provide a general methodology to analyze and evaluate these effects. Although we concentrate on the EUL for UMTS the methodology can easily accommodate other technological scenarios, e.g. LTE uplink.

The impact of relaying on inter-cell interference is a less studied topic. Due to the shorter communication ranges that a relay introduces, a mobile station (MS) needs lower transmit power for successful reception. Hence, in a system of relayenabled cells, we can expect lower inter-cell interference which in turn suggests an improvement in channel conditions, i.e. higher data rates. For example, [7] presents a case of UMTS downlink scenario with relays and inter-cell interference. The author shows, for a single MS, that sending via the relay is beneficial in terms of packet errors and delays.

In the current paper we study what are the effects of relaying on inter-cell interference and the subsequent impact on users' performance. First, the inter-cell interference pattern of a relayenabled cell is analysed using two independent approaches, i.e. by analytical calculation and by system simulation. Second, accounting for the interference, we evaluate the performance of MSs in terms of realized data rates and flow throughputs, stressing on the importance of the latter.

We consider three scheduling schemes - two relay-enabled ones and a reference scheme, which does not use a relay. The scheduling scheme influences both inter-cell interference and relaying. While relaying mainly affects interference levels, scheduling determines the fluctuation of these levels in time, see [8]. Choosing an appropriate scheduler also allows the multi-user diversity in the uplink to be used to maximize resource utilization

In summary, our most relevant findings are: (i) deploying relays reduces inter-cell interference; and (ii) relaying has implicit benefits even for users who do not use it, i.e. general cell performance improves. Additionally, we show that an analytical approach to model inter-cell interference is feasible 


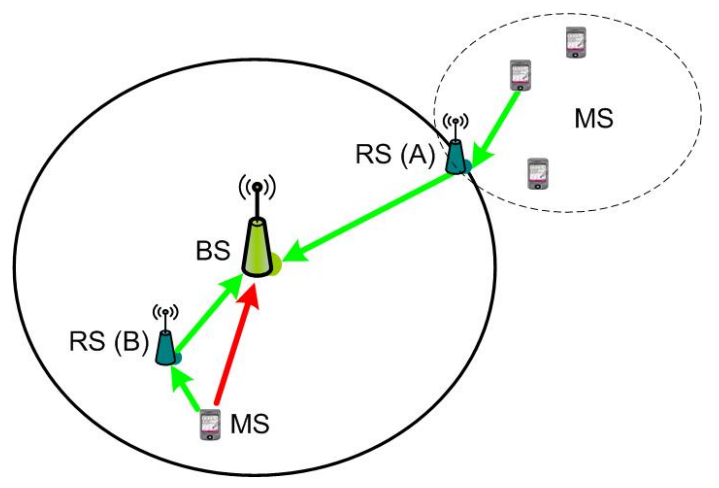

Fig. 1. Deployment of a relay station for extending coverage, e.g. RS (A), and increasing system capacity, e.g. RS(B).

and provides several advantages to simulation.

The paper continues with Section II where we briefly discuss the relaying concept and describe the scheduling schemes considered in this paper. The model description is presented in Section III. The analysis of both the inter-cell interference process and of the performance evaluation for MSs are given in Secion IV. Section V presents our findings and finally, Section VI summarizes our work and presents certain concerns about further research.

\section{RELAY-ENABLED SCHEDULING}

Since our study concentrates on EUL, several aspects of its resource management are relevant for the scheduler. The key channel resource is the total received interference budget $B$ at the base station (BS). The channel access is shared among all active users and is organized by the base station on a time scale of $2 \mathrm{~ms}$ (TTI - Transmission Time Interval). The particular assignment of TTIs over the active mobile stations depends on the scheduling scheme. Introducing a relay station (RS) requires modifications of the scheduler such that certain TTIs are dedicated to relay transmissions. First we will elaborate on the effects relaying has on MSs' performance and on transmission ranges. Second, we present the scheduling schemes of choice in this paper. Lastly, we briefly comment on the implications for inter-cell interference that relaying could cause.

\section{A. Relay Deployment}

In the case of an uplink the RS forwards data from the MSs to the BS and effectively changes (shortens) the transmission ranges. An advantage of shorter communication path is the lower power required to reach the receiver. In turn, we can expect improved performance, i.e. higher data rates, and decreased interference towards neighbour cells. However, relaying has the drawback of increased transmission time and lower maximum achievable rate due to data forwarding. Hence, whether relaying can be beneficial depends on the particular MS and its position relative to the relay and base station.

Typically the communication between MS and BS is referred to as a direct link or direct path, see [3]. In contrast,

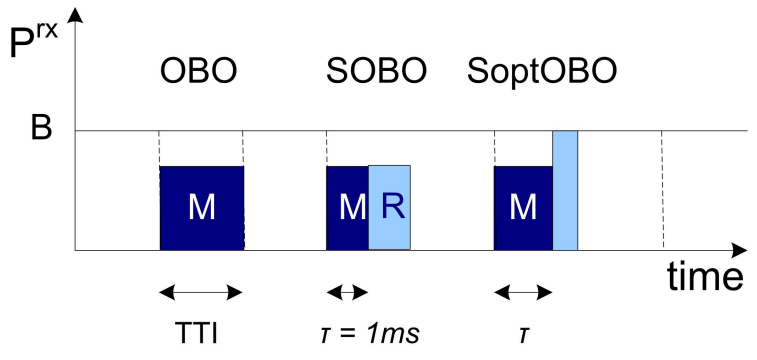

Fig. 2. Scheduling schemes: OBO, SOBO and SoptOBO

the path from MS-RS-BS we term indirect path. The indirect path consists of two sub-paths - mobile sub-path MS-RS and relay sub-path RS-BS. Each (sub-)path is characterized by a set of transmission parameters: the distance between the communicating devices $d_{z z}$, the path loss $L_{z z}$, the transmit power $P_{z z}^{t x}$, the duration of a transmission opportunity $\tau_{z z}$ and the realized data rate $r_{z z}$ during a transmission opportunity. The index $z z$ refers to the specific (sub-)path, i.e. $m s$ for the direct path MS-BS, $m r$ for the mobile sub-path MS-RS, and $r s$ for the relay sub-path RS-BS. The transmission times $\tau_{m r}$ and $\tau_{r s}$ as well as the realized data rates on the indirect path are scheduler specific and are further discussed in Section IV. We will now continue to introduce the specific scheduling schemes considered in our study.

\section{B. Scheduling Schemes}

Two relay-enabled scheduling schemes are compared in performance to a conventional non-relay scheme. All three schemes belong to the Round Robin (RR) family in which all mobile stations are given equal access to the uplink independently of their channel conditions. Since in RR schemes MSs are served one after another we use the term one-by-one (OBO) scheduler.

Studies have shown that OBO, although easy to implement, leads to underutilization when applied to UMTS uplink, e.g. [13], [14]. Battery-constrained mobile devices, especially the ones suffering from high path loss, are generally unable to use the complete channel resource on their own. However, the expectation of a relay to increase MS's utilization ability justifies our choice of OBO as scheduling strategy.

In the conventional non-relaying scheme, simply referred to as $O B O$, a MS always use the direct path with no respect to its location in the cell, see Figure 2. In OBO the duration of a single transmission opportunity equals one TTI, i.e. $\tau_{m s}=2 m s$. In each of the two relay-enabled schemes, also presented in Figure 2, a MS selects the direct or the relay path depending on which one offers higher realized rates. Given that the indirect path is chosen, both schemes schedule MS and RS transmission in the same TTI, i.e. $\tau_{m r}+\tau_{r s}=T T I$ but differ in the assigned specific times $\tau_{m r}$ and $\tau_{r s}$, see Figure 2 .

The Shared $O B O$ (SOBO) scheme divides the TTI into two equal intervals of $1 \mathrm{~ms}$ and $\mathrm{MS}$ and $\mathrm{RS}$ each receives a single interval, i.e. $\tau_{m r}=\tau_{r s}=1 \mathrm{~ms}$. In order to avoid potential problems with ever growing buffers, the realized 


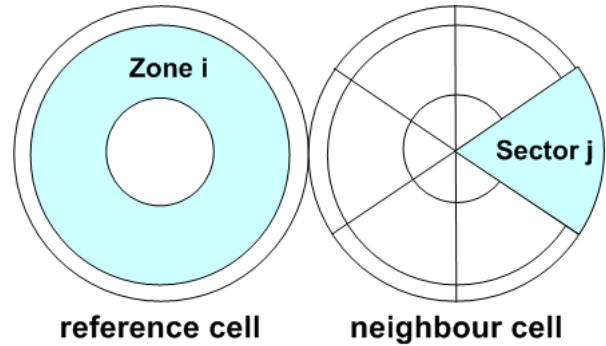

Fig. 3. Two cell model. The reference cell is divided in zones while the neighbour cell is divided in zones and sectors.

data rate on the indirect path is limited by the slower subpath. Working with fixed-length transmission opportunities ease implementation but it is not the most efficient choice when the instantaneous rates on the sub-paths differ.

In the Optimized SOBO (SoptOBO) scheme the shortcomings of SOBO are avoided by assigning different transmission opportunities $\tau_{m r}$ and $\tau_{r s}$. In particular, the times are selected such that the mobile and relay sub-paths match in transmission capacity, i.e. $\tau_{m r} r_{m r}=\tau_{r s} r_{r s}$. Individually selecting the transmission times for the sub-paths provides high order of channel utilization but unfortunately is rather challenging for implementation.

In our study we consider amplify-and-forward type of relay. Although the received noise is amplified along with the signal they are simple to deploy and do not introduce significant delays. Hence we are able to assume that the switching between receiving and transmitting at the relay station is almost instantaneous and can happen in one TTI.

\section{Intra-cell interference aspects}

In the proposed schemes relaying does not affect intra-cell interference within the cell since each MSs (on both direct and indirect path) is served individually and on its own. However, if parallel transmissions are supported relaying could lead to lower intra-cell interference due to the lower transmit powers it requires for successful reception. An interesting direction of research would be a scheme which tries to scheduler 'direct' MS simultaneously with 'indirect' MS from opposite parts of the cell. Such strategy could further decrease intra-cell interference by increasing the distance between the receiving station and the interfering station.

\section{MODEL}

The system model considered in this paper consists of two relay-enabled cells - a reference cell (RC) and a neighbour cell (NC), see Figure 3. The neighbour cell is the one generating the inter-cell interference $I_{o c}$ and the reference cell is the one where the performance of the mobile stations is evaluated. Both cells have the same radius $r$ and the inter-BS distance is $2 r$. Each cell is split up into $K$ concentric zones with equal areas, where zone $i$ is characterized by a distance $d_{i}$ to the base station and corresponding path loss denoted by $L\left(d_{i}\right)$, $i=1, \ldots, K$. Such division allows us to model the impact of the MS's location on performance. Additionally, in order to enable adequate modelling of inter-cell interference, the neighbour cell is split up into $S$ sectors, also equal sized. The intersection of zones and sectors determines a segment characterized by a distance $d_{i j}$ to the base station of the reference cell and corresponding path loss $L\left(d_{i j}\right) ; i=1, \ldots, K, j=1, \ldots, S$. The inter-cell interference in the $\mathrm{NC}$ coming from the $\mathrm{RC}$ is not modelled since [15] shows that this only complicates analysis without significantly affecting performance.

We assume that a relay is positioned always on the straight line between the BS and a MS. Although a rather optimistic assumption, it simplifies analysis and allows for a more generic evaluation, which provides us upper bound on the expected performance gains. The distance between the BS and a RS is fixed at the constant metric $d_{r s}$.

The interference budget $B$ available at the base station is the same also at the relay station and can be derived from an operator-specific noise rise target. Part of the budget is lost to interfering signals. In particular, at the $\mathrm{BS}$ of the $\mathrm{RC}$ a variable reservation $B_{o c}$ is introduced to cope with the anticipated intercell interference. $B_{o c}$ is dynamically updated to follow the changes in the actual inter-cell interference generated by the NC. We assume the updates of $B_{o c}$ to be instantaneous. The interference budget available for the service of EUL data users, i.e. available data budget $B^{\prime}$, becomes $B^{\prime}=B-B_{o c}$.

Mobile stations become active according to a Poisson process with rate $\lambda$ and are uniformly distributed over the cell. Given the equal size area assumption, the arrival rate per zone in the $\mathrm{RC}$ equals $\lambda / K$ and per segment in the $\mathrm{NC}$ equals $\lambda /(K \times S)$. Flow size is exponentially distributed with mean size $F$ (in kbits). All users have the same maximum transmit power $P_{\max }^{t x}$ but different maximum received power at the base station $P_{\max }^{r x}$ due to the zone-dependent path loss. A MS which is close to the BS and can fully utilize the channel resource $B^{\prime}$ transmits at power lower than the maximum. As no user mobility is considered, shadowing is not relevant and is not modelled.

\section{ANALYSIS}

The analysis of each of the scheduling schemes runs roughly in two steps. First, the inter-cell interference process generated by the neighbour cell is determined. Second, the performance within the reference cell is analysed. Both steps require knowledge on the dynamics within the cell, i.e. the changing number of flows. Hence, we need to model the MSs' behaviour within a cell.

\section{A. Cell Dynamics}

Cell dynamics are determined by the arrival rate of new flows and the rate at which flows are served. The service rates depend on several factors among which the distance of a MS to the base station. The discussion in this section is based on a model with cell division into $\mathrm{K}$ zones.

Our proposed analysis combines packet and flow level aspects. We start with calculation of received powers from which subsequently realized data rates can be derived. Later, we consider flow throughputs which reflect the impact of the 
changing number of active MSs. Flow dynamics, i.e. initiation and completion of flow transfers, are modelled by a continuous time Markov chains (CTMCs).

Such methodology has several advantages: (i) packet level analysis incorporates specifics of the scheduling scheme and environment; (ii) flow level analysis represents users' behaviour; (iii) working with Markov models supports fast evaluation. Furthermore, the approach is rather scalable since changes in the scheduler or the environment require only recalculations at the packet level.

1) Received Powers: Given a transmit power $P_{z z}^{t x}$, the received power $P_{z z}^{r x}$ on any communication path can be derived from the path loss $L_{z z}(d)$ as follows:

$$
P_{z z}^{r x}=\min \left(\frac{P_{z z}^{t x}}{L_{z z}(d)}, B^{\prime}\right)
$$

where $d$ is the communication path length. The index $z z=$ $(m s, m r, r s)$ denotes the (sub-)path over which the transmission is done. Note that $P_{z z}^{r x}$ and all performance parameters are location dependent, i.e. differ per zone $i$.

We apply the Okumura-Hata path loss model, namely $L(d)=123.2+10 a \log _{10}(d)$ (in $\mathrm{dB}$ ) where $a$ is the path loss exponent and $d$ is the distance in kilometres. Equation (1) is applicable to calculate received powers at the local BS or the BS of a neighbour cell.

In a SOBO scheduler the received powers at the base and relay station are the same since the indirect path transmission is limited by the slower sub-path. In SoptOBO however, according to the definition, these two powers are generally different. Note that the unbalance in received powers is compensated by the difference in transmission time.

2) Instantaneous Rate: The data rate achieved on a (sub-) path $z z$ considering only the transmission channel conditions, i.e. received power and interference, is the instantaneous rate $r_{z z}$. Hence, this is the rate realized during $\tau_{m r}$. The instantaneous rate of a particular MS is given by:

$$
\begin{gathered}
r_{z z}=\frac{R_{c h i p}}{E_{b} / N_{0}} \cdot \frac{P_{z z}^{r x}}{N+I_{o c}+(1-\omega) P_{z z}^{r x}}, \\
I_{o c}= \begin{cases}0 & \text { for } \mathrm{NC} \\
\neq 0 & \text { for RC }\end{cases}
\end{gathered}
$$

In the above equation $R_{\text {chip }}$ is the system chip rate and $E_{b} / N_{0}$ is the energy-per-bit to noise ratio. The index $z z=$ $(m s, m r, r s)$ refers to the (sub-)path. The maximum possible data rate a MS can realize is determined by the condition that the budget $B^{\prime}$ can be filled, i.e. $P_{z z}^{r x}=B^{\prime}$.

3) Effective Rate: The effective rate $r_{\text {eff }}$ accounts for the effects of relaying and is the rate realized by a MS for the duration of one TTI. On the direct path the effective rate is the same as the instantaneous, i.e. $r_{e f f}=r_{m s}$, because the entire TTI is used by the MS. On the indirect path however, due to data forwarding, the effective rate is lower than the instantaneous and depends on what part of the TTI is used by the mobile, i.e. on $\tau_{m r}$. In SOBO half the interval is used yielding $\tau_{m r}=1 / 2$ TTI. In SoptOBO $0<\tau_{m r}<T T I$ holds depending on the MS's location such that $r_{m r} \tau_{m r}=r_{r s} \tau_{r s}$ and $\tau_{m r}+\tau_{r s}=$ TTI. Given the scheduler specific time assignment policy, $r_{\text {eff }}$ can be derived from the instantaneous rate as:

$r_{e f f}= \begin{cases}r_{m s} & \text { OBO and direct path in others } \\ \min \left(r_{m r}, r_{r s}\right) * \frac{1}{2} & \text { indirect path in SOBO } \\ \frac{r_{m r} * \tau_{m r}}{\tau} & \text { indirect path in SoptOBO }\end{cases}$

4) State Dependant Throughput: Both the instantaneous and the effective rate do not account for the number $n$ of active MSs in the cell. This is why they are rather optimistic performance measures. In fact, in a Round Robin OBO scheduler, after being served a MSs might have to wait several TTIs before receiving service again. As result its actual data rate decreases. This new rate we term state-dependent throughput $R(n)$ and its dependency on $n$ is given by:

$$
R(n)=\frac{r_{e f f}}{n},
$$

5) Flow Throughput: In a real system the number of active flows continuously changes. From Equation (5) we can expect that also the state dependent throughput fluctuates in time. In order to realistically evaluate the scheduling schemes we introduce the performance measure flow throughput. Flow throughput is the data rate seen by a flow for the duration of its transfer, i.e. long-term average data rate.

A cell with dynamic flow behaviour can be very well modelled by a continuous time Markov chain. The assumptions made in Section III, namely a Poisson flow arrival process and exponentially distributed flow size, permit such mapping to be made. A state in the Markov chain is mapped to a particular distribution of MSs in the cell and an arrival/departure in the chain is analogous to a initiation/completion of a flow transfer. The transition rates of the Markov model then become:

$$
\begin{aligned}
& \left(n_{1}, \cdots, n_{i}, \cdots, n_{k}\right) \rightarrow\left(n_{1}, \cdots, n_{i}+1, \cdots, n_{k}\right) \quad \text { at rate } \lambda_{i} \\
& \left(n_{1}, \cdots, n_{i}, \cdots, n_{k}\right) \rightarrow\left(n_{1}, \cdots, n_{i}-1, \cdots, n_{k}\right) \quad \text { at rate } \frac{n_{i} R_{i}(n)}{F}(6)
\end{aligned}
$$

where $i$ indicates the zone number.

Once we have constructed the Markov model of a scheduler we can determine its steady state distribution and derive parameters such as flow throughout or mean flow transfer times. In particular, the flow throughput is given by:

$$
T h=r_{e f f} *(1-\rho)
$$

where $\rho=\sum \rho_{i}$ is the system load and $\rho_{i}=\lambda_{i} / r_{e f f, i}$ is the load in a particular zone $i$.

Note that the CTMC of the different schedulers will be different. When the form of the model allows it we find the steady state distribution by close-form equations. If the form is rather complex, we apply simulation of the Markov chain.

\section{B. Inter-cell Interference Process}

The inter-cell interference $I_{o c}$ generated by the $\mathrm{NC}$ at the base station of the RC is a stochastic process. In order to model it correctly we need knowledge on the interference values and 
the probability with which they exhibit. In this study, given the model described in Section III, we show that an analytical computation of the $I_{o c}$ distribution is feasible and has several advantages, among which faster evaluation, when compared to a simulation approach.

Let the system state $\underline{n}$ denotes the number of active MSs and their distribution over the zones (segments) of a cell. Given that we divide the NC into $K * S$ segments the NC's systems state becomes $n=\left[n_{11}, n_{12}, \cdots, n_{K S}\right]$, where $n_{i j}$ is the number of MSs in segment $i j, i=1, \cdots, K$ and $j=1, \cdots, S$.

The possible $I_{o c}$ values depend on in which segments of the NC the active MSs are located. Given a distance $d_{i j}$ and a corresponding path loss $L\left(d_{i j}\right)$, we can calculate the inter-cell interference, i.e. received power at the RC's BS, by applying Equation (1). How the probability of a particular value is determined is specific to the used approach.

The probability distribution of the inter-cell interference can be analytically computed under the assumption that the neighbour cell behaves independently of the reference cell. The latter translates to no inter-cell interference from the RC, i.e. $I_{o c}=0$. In such interference-free environment the effective rate $r_{\text {eff }}$ and the state-dependent throughput $R(n)$ depend only on known parameters, see Equations (4) and (5) respectively. In such case the probability of a particular $I_{o c}$ value is equal to the load in a segment. The probability of zero interference equals the probability of the system being empty. Hence, for the analytical approach we can write:

$$
I_{o c}= \begin{cases}0 & \text { with } \operatorname{Pr}=1-\rho \\ I_{o c, i j} & \text { with } \operatorname{Pr}=\rho_{i j}\end{cases}
$$

In a simulation approach the actual state transitions in the Markov model of the NC are generated. Knowing (i) the time spent in the state and (ii) the total simulation time, we can derive the probability distribution.

\section{Performance of Mobile Stations}

The performance of mobile stations in the reference cell is evaluated in terms of realized effective rates and flow throughputs. Both parameters depend on the inter-cell interference, which is shown by the relation of Equations (4) and (7) to Equation (2). In order to account for the changes in inter-cell interference, we introduce a new parameter at the receiver, i.e. $\mathrm{BS}$ or RS, termed reservation $B_{o c}$.

The selection of the reservation $B_{o c}$ is a rather important issue. Hypothetically $B_{o c}$ should be adapted at each change in the $I_{o c}$, i.e. $B_{o c}=I_{o c}$, leading to recalculation of the data rates, e.g. Equation (4). However, given a system state $\underline{n}$, the interference changes at a frequency of one TTI, i.e. $2 \mathrm{~ms}$ and such fast changes are impossible to account for in a real system. It is more feasible to adapt $B_{o c}$ only at state change in the $\mathrm{NC}$ while for the duration of the state the reservation stays at a fixed value. We propose two strategies towards the section of this value. In the first strategy the maximum $I_{o c}$ generated during a state $\underline{n}$ is used yielding $B_{o c}=I_{o c, \max }(\underline{n})$. In the second strategy the weighted average is taken $B_{o c}=I_{o c, a v}(\underline{n})$.
Note that, in the case of a relay-enabled scheduler, the intercell interference at the relay station generally differs from the interference received at the base station even if the state in the $\mathrm{NC}$ is the same. This is accounted for in our analysis.

The combined analysis of reference and neighbour cell results in a Markov model of the two-cells system with $(K+K * S)$ dimensions. The first $K$ dimensions correspond to the division of the RC into $K$ zones, while the second term represents the NC, i.e. $K * S$ segments. The model is rather complex due to its dependability on the particular $I_{o c}$ level, which is why we selected evaluation by simulation.

\section{NumericAl RESUlts}

After we present the general parameters set up we continue to discuss two groups of results. First, for each scheduler the combined impact of relaying and scheduling on the intercell interference are presented. Also here the two proposed approaches to model inter-cell interference, i.e. analytical and by simulation, are compared. Second, we evaluate the performance of MSs for the three schemes at both packet and flow level in terms of effective data rates and flow throughput respectively.

\section{A. Parameters Settings}

For the analytical approach to determine the inter-cell interference from the NC we use $\mathrm{K}=200$ zones and $\mathrm{S}=360$ sectors. For the simulation model we use smaller number of zones and sectors in order to keep calculation time acceptable, i.e. $K=20$ zones and $S=36$ sectors. Both cells have the same cell radius $r=2 \mathrm{~km}$. The distance $d_{r s}$ of a relay station is set at $1 \mathrm{~km}$ from the base station.

The interference budget for relay and base station is $B=$ $8.09 e^{-14}$ Watt, whcih is derived by a noise rise or $6 \mathrm{~dB}$. In the calculation of the instantaneous rate we have used a system chip rate of $3840 \mathrm{kchips} / \mathrm{s}$, a thermal noise level $N=2.7 e^{-14}$ Watt, energy per-bit to noise ratio $E_{b} N_{o}=5 \mathrm{~dB}$ and the transmit power of MS and RS both is $P_{\max }^{t x}=0.125 \mathrm{Watt}$. The mean file size is set to $F=1000 \mathrm{kbit}$. We do not consider self interference, i.e. $\omega=1$. The applied call arrival rate is set to $\lambda=0.5$ users-per-sec for both cells, which lead to a cell load of $\rho=0.82$ in the case of OBO scheduler. For the relay enabled scenario the load of the cell using the same arrival rate is expected to be lower due to higher data rates.

\section{B. Inter-cell Interference Process}

The discussion on the $I_{o c}$ levels is based on results generated by only analytical modelling. Later in Section V-B2 we present comparison with the results yielded by simulation.

1) Inter-cell Interference Levels: The unique CDF graph of the inter-cell interference process for each of the scheduling schemes is presented in Figure 4. The maximum observed $I_{o c}$ for the SOBO and SoptOBO schemes is lower than the value for OBO because MSs located at the cell edge transmit through the relay and use lower transmit power. For the same reason, in the relay-enabled schemes, low interference values are more probable to occur. 


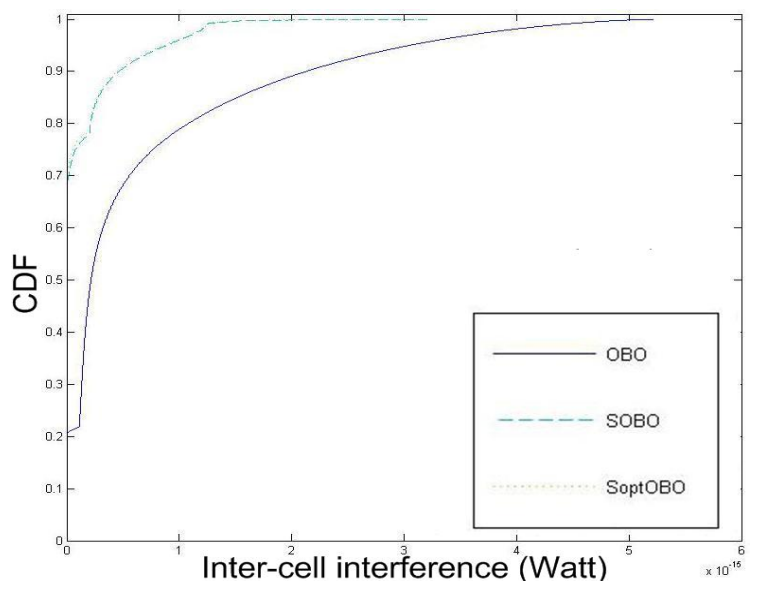

Fig. 4. CDF of the inter-cell interference process for each of the scheduling schemes.

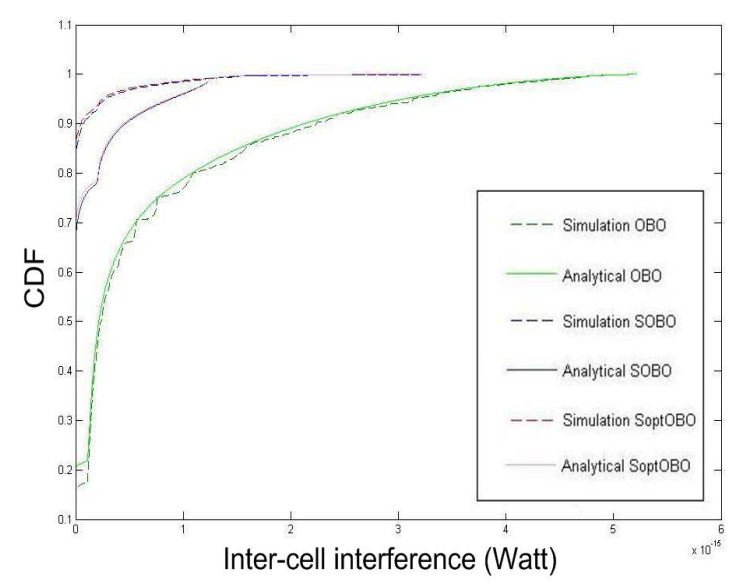

Fig. 5. Comparison of the CDFs for the inter-cell interference generated analytically or by simulation.

We have taken into account also the empty system state in which no interference is generated. Observe that the probability for zero interference for the OBO scheduler is more than three times lower compared to SOBO and SoptOBO. This is due to the fact that MSs under relay-enabled schedulers have higher transfer rates and lower transmission times. Further, the difference in the CDFs of SOBO and SoptOBO is hardly observable.

The particular contribution of each MS to the $I_{o c}$ for OBO and SOBO is presented in Figures 6(a) and 6(b) respectively. The graphs plot the $I_{o c}$ at the $\mathrm{BS}$ of the RC as a function of the location of a MS in the NC. The increase in the graphs corresponds to the $\mathrm{NC}$ area facing the $\mathrm{RC}$. The figure confirms our observations that an SOBO scheme generates lower maximum $I_{o c}$, i.e. $I_{\max }=3.2 e^{-15} \mathrm{Watt}$, than a $\mathrm{OBO}$ scheme, i.e. $I_{\max }=5.2 e^{-15}$ Watt . The maximum interference level is generated by a MS at the cell edge of the NC closest to the RC. Such MSs are relatively far from their serving base station in the $\mathrm{NC}$ and need to transmit at maximum power, i.e. $P_{\max }^{t x}=0.125 \mathrm{Watt}$.

In Figure 6(b) when moving away from the centre of the cell

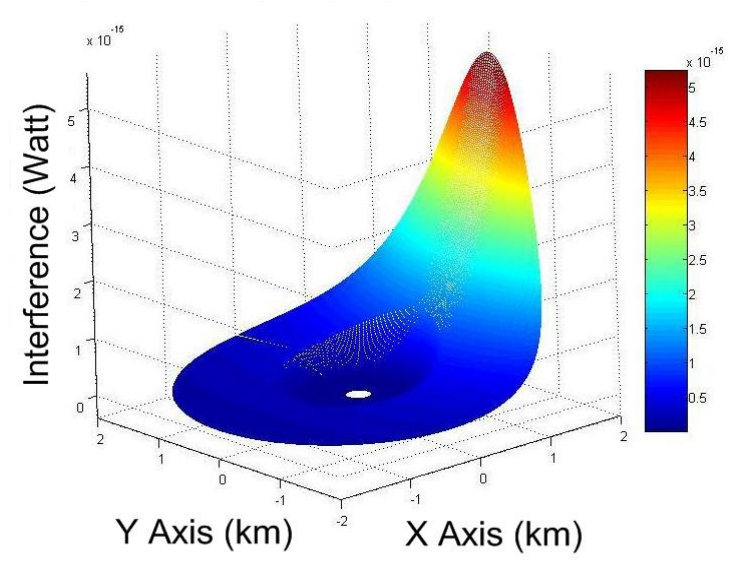

(a) OBO scheduler

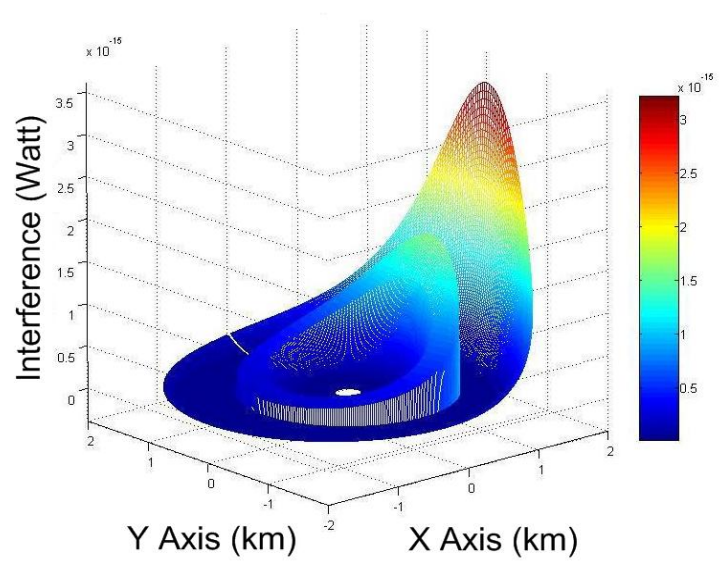

(b) SOBO scheduler

Fig. 6. Spatial representation of the individual contribution a MS has to the inter-cell interference depending on its location in the cell. The results for: (a) OBO and (b) SOBO scheme are presented.

a sudden drop in the inter-cell interference is observed. At that moment relaying becomes beneficial and a MS needs lower transmit power for successful reception thus decreasing $I_{o c}$. Subsequently, as the distance between MS and RS increases the $I_{o c}$ is again on the rise.

The specific impact of the relay stations on the inter-cell interference can be observed in Figure 4. The CDF for the SOBO and SoptOBO schedulers does not change smoothly. The part of the graph enclosed by the inflex points reflects the impact of the relay station. Each relay serves several MSs and thus $I_{o c}$ values generated by the relays appear more often.

2) Process modelling: We will now compare the inter-cell interference patterns generated by two independent approaches - analytical and simulation. For the ease of notation we will refer to the $\mathrm{CDF}$ generated via analytical computation as $C D F$ an and to the CDF generated during simulation as CDF-sim.

The CDFs for both approaches and for each of the three schedulers are presented in Figure 5. The general impression is that the graphs of the CDF-an and CDF-sim, taken per scheduler, lay very close to each other. The maximum values of the inter-cell interference registered by the CDF-an and 


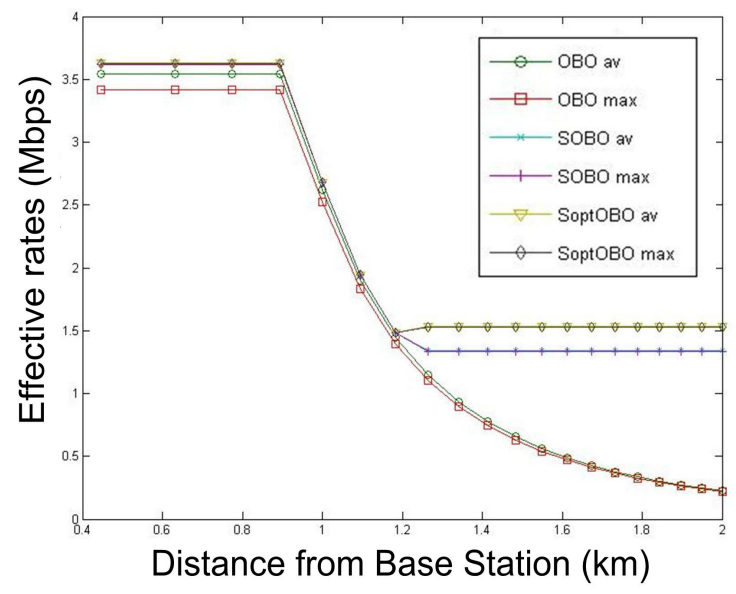

Fig. 7. Performance evaluation on the packet level in terms of effective rates.

CDF-sim coincide as well. The analytical approach however exhibits several advantages.

First, the analytical approach is faster since it requires only simple computations. Second, because it is faster, the analytical approach allows modelling of the $\mathrm{NC}$ in finer granularity, e.g. division in larger number of segments. For example, we were able to generate results with cell division in $200 * 360=72000$ segments within several minutes. A simulation with only $20 * 36=720$ segments took about ten times longer, i.e. about $40 \mathrm{~min}$.

The impact of the granularity level is visible in Figure 5. The finer the granularity the larger the number of segments in the cell model and the larger the set of possible $I_{o c}$ levels. Since the simulation approach supports less fine granularity, the CDF-sim graphs are characterised by a discrete, stepwise form which is best observed for the OBO scheme. With OBO most MSs use the maximum transmit power such that the interference level depends only on the distance, see Equation (1). In a relay-enabled scheme however, the applied transmit power is also a factor which contributes to a larger diversity in the possible interference levels.

\section{Performance of Mobile Stations}

1) Effective Rate: Figure 7 presents the results for the effective rate of the three schedulers (OBO, SOBO and SoptOBO) for the two $B_{o c}$ reservation strategies - at average and maximum $I_{o c}$ value. As we expected relaying increases realized data rates. In particular, on the indirect path SoptOBO outperforms the SOBO scheme due to its better flexibility in channel assignment as a function of MS's transmit capacity. More importantly, relay-enabled schedulers register better performance even for MSs using the direct path, i.e. see distance ranges up to $1 \mathrm{~km}$ in Figure 7 . This is a consequence of the lower $I_{o c}$, see Section V-B1 and Equation 2.

The impact of the $B_{o c}$ reservation choice is presented in Figure 7. For the OBO scheme performance differs significantly $3.55 \mathrm{Mbps}$ for average value of $I_{o c}$ against the lower $3.41 \mathrm{Mbps}$ with reservation at maximum value. On the contrary, SOBO

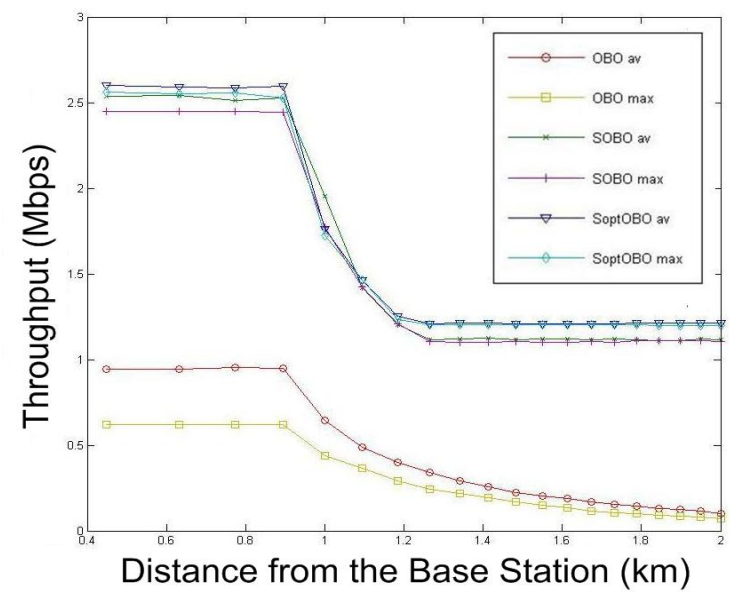

Fig. 8. Performance evaluation on the flow level in terms of flow throughput.

and SoptOBO show negligible change in performance.

Additionally, several general observations can be made. The first (higher) flat section of the graphs in Figure 7 corresponds to MSs close enough to the BS to fill up the available data budget on their own, i.e. $P_{m s}^{r x}=B^{\prime}$. With the chosen parameter settings, the maximum distance for which $P_{m s}^{r x}=B^{\prime}$ holds is $0.9 \mathrm{~km}$, what Figure 7 indicates as well. Further increase in the distance leads to degradation in the date rates. The second (lower) flat section for the graphs of SOBO and SoptOBO is determined by the fact that all MSs served via a relay are limited in transmission by the relay. The realized rates are lower than the maximum even if the budget can be filled due to longer transmission times.

2) Flow Throughput: Figure 8 shows a comparison between the achieved throughput for OBO, SOBO and SoptOBO scheme. SoptOBO shows the best performance, followed by SOBO and OBO with the worst performance. All general observations made for the effective data rates hold for the flow throughputs as well.

On the flow level, the big performance difference between $\mathrm{OBO}$ and the relay-enabled schemes become even more distinguishable, especially for MSs which do not use the relay. The faster service offered by the relay to remote MSs translates to lower average number of active flows from which all MSs, independently of location, benefit, see Equation (5). These results strongly support our claim that evaluation at flow level is crucial for understanding the complex effects of relaying.

All results show that both effective rates and flow throughput of SOBO and SoptOBO schemes are less sensitive, if not robust, to the $B_{o c}$ reservation strategy. Therefore relayenabled schemes become attractive also from practical point of view, e.g. implementation. In the case of OBO however, due to the big difference in performance further research on the impact of the reservation strategy on resource utilization and outage probability is recommended. Possibly selecting a percentile of the $I_{o c}$ distribution to serve as $B_{o c}$ might prove better. Unfortunately such approach is more challenging to 
implement and requires detailed knowledge of the inter-cell interference in real time.

\section{CONCLUSION}

We discussed the combined impact relaying and intercell interference have on the performance of mobile stations in the context of EUL for UMTS. For evaluation purposes we considered the inter-cell interference pattern as well as performance measures such as realized data rates and flow throughputs. In order to examine the impact of relaying we compared two relay-enabled scheduling schemes to a reference scheme which does not make use of relaying.

Our results indicate that relaying successfully decreases inter-cell interference levels which in turn leads to improved performance for the users. Interestingly, users who do not use the relay also gain from relaying. The trend is already observable for the realized data rates but becomes particularly so when flow throughputs are compared. Hence, our conclusion is that the flows' behaviour, i.e. flow initiation and termination, is crucial for performance and needs to be taken into account during evaluation. Additionally, we show that an analytical approach towards the generation of the inter-cell interference pattern exhibits several benefits, e.g. speed, to a simulation one.

As topics for further study we propose to evaluate the effects several relay stations have on each other when sending in parallel, i.e. intra-cell interference. Such research questions are rather interesting for deployment. Designing a smart interference-aware scheduler which tries to maximize capacity by scheduling users depending on the transmissions in neighbour cells is also an attractive topic.

\section{REFERENCES}

[1] G. T. 25.308, "High Speed Downlink Packet Access (HSDPA); Overall Description."

[2] G. T. 25.309 "FDD Enhanced Uplink; Overall Description."

[3] T. Beniero, S. Redana, J. Hamalainen, and B. Raaf, "Effect of relaying on coverage in 3GPP LTE-advanced," 2009.

[4] R. Irmer and F. Diehm, "On coverage and capacity of relaying in lteadvanced in example deployments," in Personal, Indoor and Mobile Radio Communications, 2008. PIMRC 2008. IEEE 19th International Symposium on, Sept. 2008, pp. 1-5.

[5] D. C. Dimitrova, H. van den Berg, and G. Heijenk, "Performance of relay-enabled uplink in cellular network - a flow level analysis.' ICUMT'09, St Petersburg, Russia, 2009.

[6] E. Reetz, R. Hockmann, and R. Tonjes, "Performance study on cooperative relaying topologies in beyond $3 \mathrm{~g}$ systems," in ICT-MobileSummit '08, 2008.

[7] M. Umlauft, "Relay devices in umts networks - effects on," in Proceedings of the Fifth Annual Mediterranean Ad Hoc Networking Workshop (Med-Hoc-Net 2006), 2006.

[8] D. C. Dimitrova, H. van den Berg, G. Heijenk, and R. Litjens, "Impact of inter-cell interference on flow level performance of scheduling schemes for yhe umts eul." WiMob'08, Avignon, France, 2008.

[9] A. Bel and G. S.-G. Jose Lopez Vicario, "The benefits of relay selection in wimax networks," in ICT-MobileSummit '08, 2008.

[10] J. Vidal, O. Munoz, A. Agustin, E. Calvo, and A. Alcon, "Enhancing 802.16 networks through infrastructure-based relays," in ICTMobileSummit '08, 2008.

[11] S. W. Peters, A. Y. Panah, K. T. Truong, and R. W. Heath, "Relay architectures for 3gpp lte-advanced," EURASIP J. Wirel. Commun Netw., vol. 2009, pp. 1-14, 2009.

[12] R. Schoenen, R. Halfmann, and B. Walke, "An fdd multihop cellular network for 3gpp-lte,” May 2008, pp. 1990-1994.
[13] H. Holma and A. Toskala, HSDPA/HSUPA for UMTS. John Wiley \& Sons Ltd, 2006.

[14] C. Rosa, J. Outes, T. Sorensen, J. Wigard, and P. Mogensen, "Combined time and code division scheduling for enhanced uplink packet access in WCDMA.” IEEE VTC '04 (Fall), Los Angeles, USA, 2004.

[15] D. C. Dimitrova, H. van den Berg, and G. Heijenk, "Scheduler dependent modeling of inter-cell interference in UMTS EUL." NGMAST '09, Cardiff, Wales, UK, 2009. 